\title{
Social Anxiety and Anger Identification
}

\section{Bubbles Reveal Differential Use of Facial Information With Low Spatial Frequencies}

\author{
Oliver Langner, Eni S. Becker, and Mike Rinck \\ Behavioural Science Institute, Radboud University Nijmegen
}

\begin{abstract}
We investigated the facial information that socially anxious and nonanxious individuals utilize to judge emotions. Using a reversed-correlation technique, we presented participants with face images that were masked with random bubble patterns. These patterns determined which parts of the face were visible in specific spatial-frequency bands. This masking allowed us to establish which locations and spatial frequencies were helping participants to successfully discriminate angry faces from neutral ones. Although socially anxious individuals performed as well as nonanxious individuals on the emotion-discrimination task, they did not utilize the same facial information for the task. The fine details (high spatial frequencies) around the eyes were discriminative for both groups, but only socially anxious participants additionally processed rough configural information (low spatial frequencies).
\end{abstract}

Cognitive theories suggest that social phobia and social anxiety are marked by altered processing of social information (Rapee \& Heimberg, 1997). Supporting evidence comes from research demonstrating attentional biases toward threatening stimuli, especially angry faces, in socially anxious individuals (Heinrichs \& Hofmann, 2001). However, social phobia and social anxiety are not accompanied by better detection of negative facial expressions (Schofield, Coles, \& Gibb, 2007) or biases to evaluate facial expressions more negatively (Philippot \& Douilliez, 2005). These results are at odds with cognitive models of anxiety, which postulate that negative appraisal of social stimuli plays a crucial role in attentional biases (Beck \& Clark,

Address correspondence to Oliver Langner, Behavioural Science Institute, P.O. Box 9104, 6500 HE Nijmegen, The Netherlands, e-mail: o.langner@psych.ru.nl.
1997). However, these findings do not imply that socially anxious individuals process visual social information in the same way as nonanxious individuals. We propose that anxious and nonanxious individuals use different visual information to detect angry facial expressions, even though they may not differ in detection performance.

Evidence for differences in selecting facial information comes from eye movement studies showing altered viewing patterns in anxious individuals when looking at angry faces: Whereas nonanxious individuals mainly fixated eyes and nose, socially anxious individuals avoided these facial features and instead scanned less-informative regions (Horley, Williams, Gonsalvez, \& Gordon, 2004). Therefore, we hypothesized that socially anxious individuals utilize information from facial regions other than the eyes-nose triangle.

In addition to location, spatial frequency is another important dimension of facial information (Morrison \& Schyns, 2001). Low spatial frequencies (LSFs) represent crude configural information, whereas high spatial frequencies (HSFs) code details like exact contours (Fig. 1). Distinguishing LSFs from HSFs is crucial because LSF information seems to be important mainly for decoding emotional expressions, and HSF information seems to be important for decoding identity of faces (Alorda, SerranoPedraza, Campos-Bueno, Sierra-Vázquez, \& Montoya, 2007; Winston, Vuilleumier, \& Dolan, 2003). For example, the amygdala responds vigorously to fearful LSF expressions, but is "blind" to fearful HSF expressions (Vuilleumier, Armony, Driver, \& Dolan, 2003). Because the amygdala is particularly sensitive to LSF facial information (Vuilleumier \& Pourtois, 2007), and neuroimaging studies suggest that the amygdala is hyperresponsive in anxiety (Phan, Fitzgerald, Nathan, \& Tancer, 2006; Stein, Goldin, Sareen, Eyler Zorrilla, \& Brown, 2002), we hypothesized that socially anxious individuals should exhibit a bias toward processing LSF facial information. 


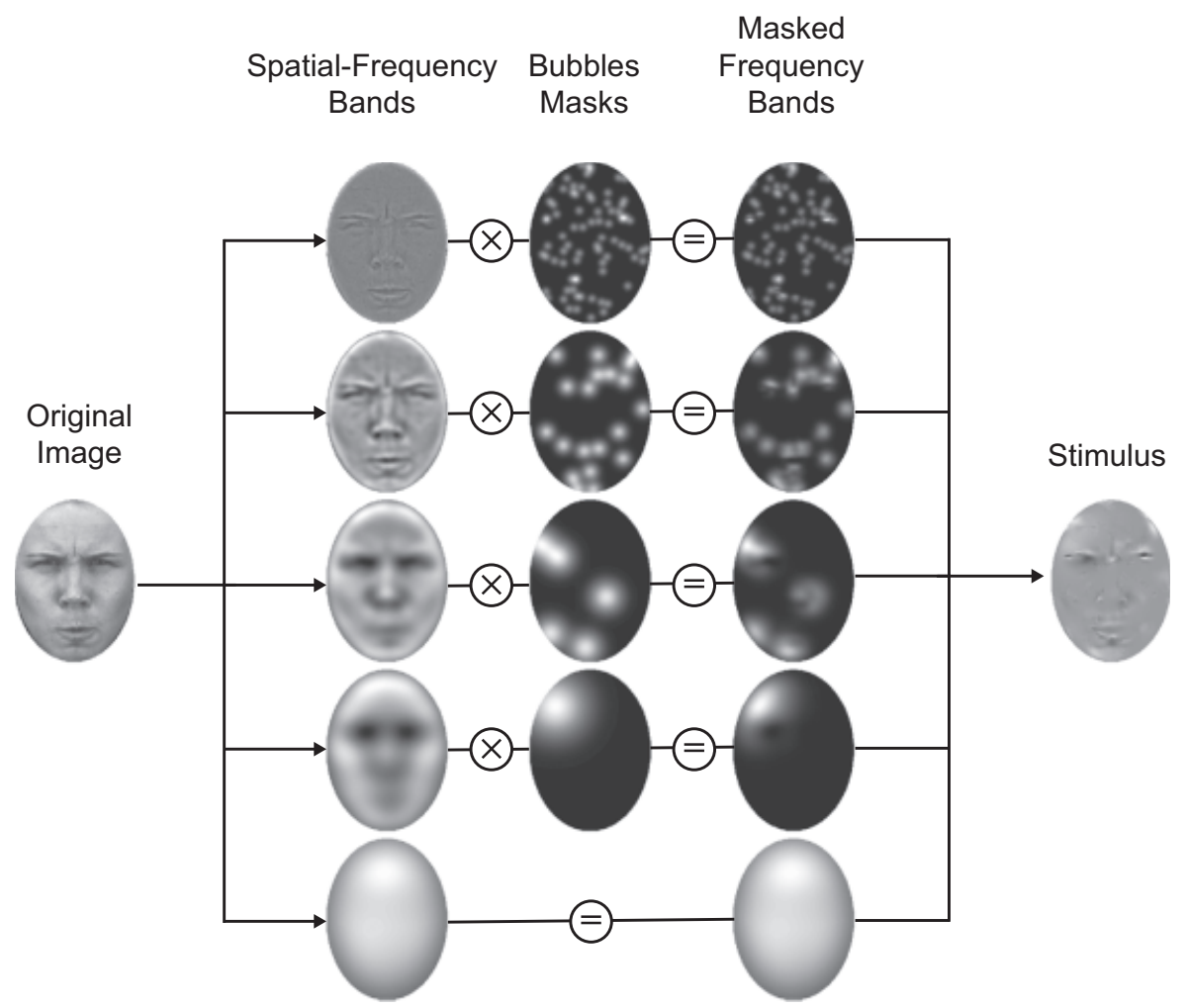

Fig. 1. Example of how the stimuli in this study were constructed. First, the original image was decomposed into five frequency bands. Then, the first four frequency bands were masked by randomly placed bubbles, such that facial information was revealed only through the bubbles. The fifth frequency band, which contained hardly any facial information, was kept as background, without alteration. Finally, the information revealed in all five bands after masking was recombined to produce the stimulus image.

We used the Bubbles paradigm (Gosselin \& Schyns, 2001), a method for identifying which image locations and spatial frequencies are critical for discriminating between stimuli; in this study, participants discriminated between neutral and angry faces (Schyns, Bonnar, \& Gosselin, 2002; Spezio, Adolphs, Hurley, \& Piven, 2007). Bubbles are two-dimensional Gaussian masks that reveal only small circular image parts, like punch holes in a sheet of paper (see Fig. 1).

\section{METHOD}

Forty-one female students from Radboud University Nijmegen participated in the study. Twenty-two participants had low social anxiety (mean age $=22$ years, $S D=4.8$ years), and 19 participants had high social anxiety (mean age $=20$ years, $S D=1.5$ years), as measured by the Liebowitz Social Anxiety Scale (Liebowitz, 1987). Mean scores on this scale were $9.7(S D=3.1)$ for low-anxiety participants and $32.8(S D=5.6)$ for high-anxiety participants.

In 880 forced-choice trials, participants judged whether masked faces, which were presented for $2 \mathrm{~s}$, showed a neutral or angry expression. We used 80 images (20 male faces with neutral expressions, 20 male faces with angry expressions, 20 female faces with neutral expressions, and 20 female faces with angry expressions) from the Karolinska Directed Emotional Faces (Lundqvist, Flykt, \& Öhman, 1998). Position of the eyes, nose, and mouth were normalized, and ears and hair were hidden by an oval vignette. For each trial, a new stimulus was generated (see Fig. 1): First, each image was split into five frequency bands, each containing a limited range of spatial frequencies from the original image. The level of detail ranged from fine contours in the highest frequency band to very broad configural features in the lowest frequency band. Next, the first four frequency bands were masked by bubble masks, such that only information from the randomly placed bubbles was revealed. The fifth band remained completely visible as background because it contained hardly any facial information. Finally, all masked bands were recombined to form the actual stimulus, in which information was visible only through the bubbles (Gosselin \& Schyns, 2001). Stimuli measured $16.4^{\circ} \times 12^{\circ}$ visual angle $(418 \times 302$ pixels $)$. In addition to tracking eye movement, this method identifies the image locations and the spatial frequencies at these locations that are informative for the task.

Because of the random placement of bubbles, some stimulus parts contained no information (no bubble), some showed information from one spatial-frequency band (single bubble in a band), and some showed a mixture of several frequency bands 
(overlapping bubbles in several bands). The relative bubble size increased from the first band to the fourth band, matching the increasing size of the informative features. So that the total area revealed would be the same for all frequency bands, the highest frequency band contained many small bubbles, and lower frequency bands contained fewer, but bigger, bubbles.

During the first 240 trials, the number of bubbles shown in each stimulus was adjusted by a staircase procedure, so that less image area was revealed after correct responses and more image area was revealed after incorrect responses. The staircase design (1-up, 4-down; Kaernbach, 1991) ensured that the individual number of bubbles required for a performance of about $80 \%$ correct responses could be estimated for each participant. In the remaining trials, all stimuli contained this number of bubbles.

\section{RESULTS}

Because we adjusted the number of bubbles to ensure an $80 \%$ correct response rate, the groups did not differ in the percentage of correct responses $(M=82 \%$ correct, $S D=4.7 \%), t(39)=$ $0.3, p=.74$, or in the sensitivity index $d^{\prime}(M=2.0, S D=0.3)$, $t(39)=0.4, p=.68$. There were no group differences in the number of bubbles presented after the staircase procedure (average $=57.0$ bubbles, $S D=20.7), t(39)=0.1, p=.94$, or in the response-bias index $c(M=-0.03, S D=0.34), t(39)=0.1$, $p=.94$. Thus, the two groups received a similar amount of visual information and performed equally well on the task.

To identify facial features that helped participants discriminate expressions, we computed discrimination images for each group and each frequency band (Gosselin \& Schyns, 2001). First, we determined the average visibility of information for correct and incorrect trials separately. For each image pixel, we averaged the bubble-mask weights (see Fig. 1) of all correct trials and subtracted the result from the average bubble-mask weights of all incorrect trials. Average bubble-mask weights ranged from 0 (no information visible) to 1 (information fully visible). Stated formally, for each pixel, we computed the probability that information was displayed through the bubbles, separately for correct and incorrect responses. A large difference between these probabilities indicated that the corresponding pixel was highly important for the expression judgment, so that participants systematically responded correctly if information was visible in that pixel, and incorrectly if not. To identify significantly discriminative areas, we subjected these difference images to cluster tests that specified how many adjacent pixels had to be significant for the finding not to be due to chance. Cluster tests are commonly used for testing many dependent variables that are correlated with their neighbors, like image pixels or brain voxels (Chauvin, Worsley, Schyns, Arguin, \& Gosselin, 2005). We used a desired significance value of .001 and a threshold $t$ value of 2.5 for the cluster tests. The tests resulted in minimal cluster sizes of $71,182,293$, and 397 pixels for the first through fourth frequency bands, respectively. Only clusters with more significant pixels than the minimal size were labeled significant (see Details of Analysis in the Supporting Information available on-line; see p. XXX). Discrimination images for both groups are shown in Figure 2.

Additional cluster tests were calculated to test group effects. First, we subtracted the difference images of one group from the difference image of the other group. Large group differences indicated image locations that were discriminative for one group only. Then, we performed cluster tests on these differences. Image areas that were significantly more discriminative in one group than in the other are tinted red in Figure 2. For both groups, HSF information around the eyes was discriminative for judging emotion. Unlike the nonanxious participants, who did not use much LSF information, the highly anxious also relied on LSF information from eyes, nose, and mouth (see right-most images of Fig. 2).

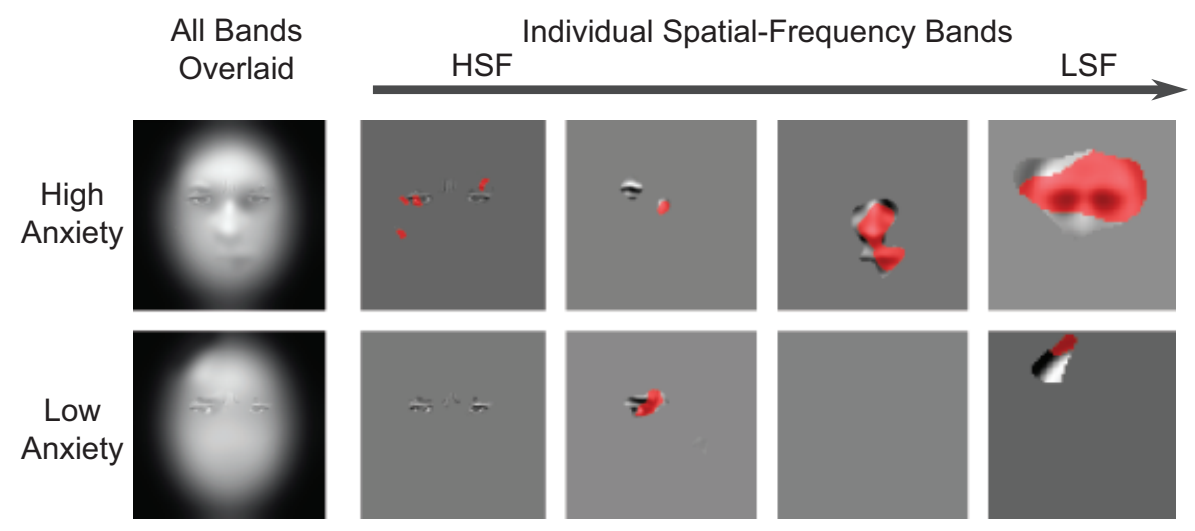

Fig. 2. Clusters of information used by participants with high (top row) and low (bottom row) social anxiety to judge emotion. In each row, the left-most image shows the discriminative information from all frequency bands superimposed. The images to the right show the discriminative information from each of the masked individual frequency bands, ranging from high spatial frequency (HSF) to low spatial frequency (LSF). Areas shown in red were significantly more discriminative for the indicated group than for the other group. 


\section{DISCUSSION}

The results demonstrate systematic differences in which information was most discriminative for participants with high and low social anxiety when discriminating angry from neutral faces. Socially anxious participants used LSF information from eyes, nose, and mouth regions that was not used by nonanxious participants. This higher sensitivity of socially anxious participants to LSF information might be due to a hyperresponsive amygdala, which is known to be particularly sensitive to LSF information. This possibility should be addressed in future research.

Although socially anxious participants used more image regions and spatial frequencies overall, they did not perform better than nonanxious participants. This result is in accordance with earlier studies that found no differences between nonanxious participants and socially anxious or socially phobic participants in the categorization or detection of emotional expressions (Philippot \& Douilliez, 2005; Schofield et al., 2007). However, our result also demonstrates that this is not the whole story: Socially anxious participants systematically used additional visual information to reach the same performance level as nonanxious participants. This finding implies that socially anxious individuals might compensate for weaker performance in decoding emotions from HSF information by utilizing LSF information. Alternatively, socially anxious individuals might be better than nonanxious individuals at decoding emotions from LSF information alone, and thus might not have to rely exclusively on HSF information. Because our stimuli always contained information from all frequency bands, we cannot conclude whether the socially anxious participants' preference for LSF information was based on better performance for LSF information, impaired processing of HSF information, or both. Future studies should address this question by presenting stimuli with HSF or LSF information alone.

For both groups, detailed HSF information around the eyes was highly discriminative. This result seems at odds with those of Horley et al. (2004), who reported that social phobics avoided looking at the eyes. However, our task probably did not elicit avoidance because no intact faces were shown. Moreover, because all stimuli were heavily masked, participants were forced to use all information available to perform successfully.

For socially anxious individuals, LSF information around the eyes was also highly discriminative; this finding indicates a clear processing bias toward LSF. Although also at odds with the findings of Horley et al. (2004) at first sight, our results suggest an interesting alternative interpretation of their findings: Because LSF information can be discriminated outside the visual focus (Virsu \& Rovamo, 1979), socially anxious individuals might not need to directly look at the eyes to identify signs of threatening facial expressions. The use of nose and mouth features by socially anxious individuals is in accordance with results from earlier eye-tracking studies (Horley et al., 2004), but our study extends those results by showing that only LSF information of those facial features is highly discriminative.
Another question is why nonanxious participants did not use information from mouth regions in our study, but did use information from these areas in a similar task studied by Schyns et al. (2002). This may be due to the fact that Schyns et al. compared neutral faces with happy faces, for which the mouth seems the most discriminative feature. Future research should investigate whether the processing bias for LSF information observed here also applies to other emotional expressions.

Although the cognitive processes involved in the current categorization task differ from those in attentional-bias tasks, the observed processing bias might play an important role in automatic attentional processes. Stronger reliance on LSF features might correspond to higher amygdala sensitivity in social anxiety being mediated by a direct subcortical pathway (LeDoux, 2000; Öhman, 2005). Through that fast, direct route, LSF information of threatening facial expressions could elicit early amygdala activation, which in turn could act as a bias signal and guide the allocation of spatial attention (Johnson, 2005). Therefore, future studies should address whether attentional biases for threatening facial expressions in social anxiety are limited to LSFs.

In conclusion, the Bubbles paradigm revealed, under highly restrictive conditions, that individuals with high and low social anxiety processed different spatial frequencies to discriminate emotions, a finding that had not yet been detected by traditional measures like categorization ratings or eye tracking. However, whether the observed LSF bias generalizes to everyday face processing remains to be studied.

Acknowledgments - This study was supported by a grant from the Behavioural Science Institute, Radboud University Nijmegen. We thank Daphne Fonteyne for data acquisition and Ron Dotsch, Paul Eling, Ad van Knippenberg, and Daniel Wigboldus for helpful comments on the manuscript.

\section{REFERENCES}

Alorda, C., Serrano-Pedraza, I., Campos-Bueno, J.J., Sierra-Vázquez, V., \& Montoya, P. (2007). Low spatial frequency filtering modulates early brain processing of affective complex pictures. Neuropsychologia, 45, 3223-3233.

Beck, A., \& Clark, D. (1997). An information processing model of anxiety: Automatic and strategic processes. Behaviour Research and Therapy, 35, 49-58.

Chauvin, A., Worsley, K.J., Schyns, P.G., Arguin, M., \& Gosselin, F. (2005). Accurate statistical tests for smooth classification images. Journal of Vision, 5, 659-667.

Gosselin, F., \& Schyns, P.G. (2001). Bubbles: A technique to reveal the use of information in recognition tasks. Vision Research, 41, 2261-2271.

Heinrichs, N., \& Hofmann, S.G. (2001). Information processing in social phobia: A critical review. Clinical Psychology Review, 21, 751-770.

Horley, K., Williams, L.M., Gonsalvez, C., \& Gordon, E. (2004). Face to face: Visual scanpath evidence for abnormal processing of 
facial expressions in social phobia. Psychiatry Research, 127, $43-53$.

Johnson, M.H. (2005). Subcortical face processing. Nature Reviews Neuroscience, 6, 766-774.

Kaernbach, C. (1991). Simple adaptive testing with the weighted updown method. Perception \& Psychophysics, 49, 227-229.

LeDoux, J.E. (2000). Emotion circuits in the brain. Annual Review of Neuroscience, 23, 155-184.

Liebowitz, M. (1987). Social phobia. Modern Problems in Pharmacopsychiatry, 22, 141-173.

Lundqvist, D., Flykt, A., \& Öhman, A. (1998). The Karolinska Directed Emotional Faces-KDEF. Solna, Sweden: Karolinska Institutet, Psychology Section, Department of Clinical Neuroscience.

Morrison, D.J., \& Schyns, P.G. (2001). Usage of spatial scales for the categorization of faces, objects, and scenes. Psychonomic Bulletin \& Review, 8, 454-469.

Öhman, A. (2005). The role of the amygdala in human fear: Automatic detection of threat. Psychoneuroendocrinology, 30, 953-958.

Phan, K., Fitzgerald, D., Nathan, P., \& Tancer, M. (2006). Association between amygdala hyperactivity to harsh faces and severity of social anxiety in generalized social phobia. Biological Psychiatry, $59,424-429$.

Philippot, P., \& Douilliez, C. (2005). Social phobics do not misinterpret facial expression of emotion. Behaviour Research and Therapy, 43, 639-652.

Rapee, R.M., \& Heimberg, R.G. (1997). A cognitive-behavioral model of anxiety in social phobia. Behaviour Research and Therapy, 35, 741-756.

Schofield, C.A., Coles, M.E., \& Gibb, B.E. (2007). Social anxiety and interpretation biases for facial displays of emotion: Emotion detection and ratings of social cost. Behaviour Research and Therapy, 45, 2950-2963.

Schyns, P.G., Bonnar, L., \& Gosselin, F. (2002). Show me the features! Understanding recognition from the use of visual information. Psychological Science, 13, 402-409.

Spezio, M.L., Adolphs, R., Hurley, R.S.E., \& Piven, J. (2007). Analysis of face gaze in autism using "Bubbles." Neuropsychologia, 45, $144-151$.
Stein, M.B., Goldin, P.R., Sareen, J., Eyler Zorrilla, L.T., \& Brown, G.G. (2002). Increased amygdala activation to angry and contemptuous faces in generalized social phobia. Archives of General Psychiatry, 59, 1027-1034.

Virsu, V., \& Rovamo, J. (1979). Visual resolution, contrast sensitivity, and the cortical magnification factor. Experimental Brain Research, 37, 475-494.

Vuilleumier, P., Armony, J.L., Driver, J., \& Dolan, R.J. (2003). Distinct spatial frequency sensitivities for processing faces and emotional expressions. Nature Neuroscience, 6, 624-631.

Vuilleumier, P., \& Pourtois, G. (2007). Distributed and interactive brain mechanisms during emotion face perception: Evidence from functional neuroimaging. Neuropsychologia, 45, 174-194.

Winston, J.S., Vuilleumier, P., \& Dolan, R.J. (2003). Effects of lowspatial frequency components of fearful faces on fusiform cortex activity. Current Biology, 13, 1824-1829.

(RECEIVED 3/15/08; REVISION ACCEPTED 11/19/08)

\section{SUPPORTING INFORMATION}

Additional Supporting Information may be found in the on-line version of this article:

\section{Details of Analysis}

Please note: Wiley-Blackwell is not responsible for the content or functionality of any supporting materials supplied by the authors. Any queries (other than missing material) should be directed to the corresponding author for the article. 